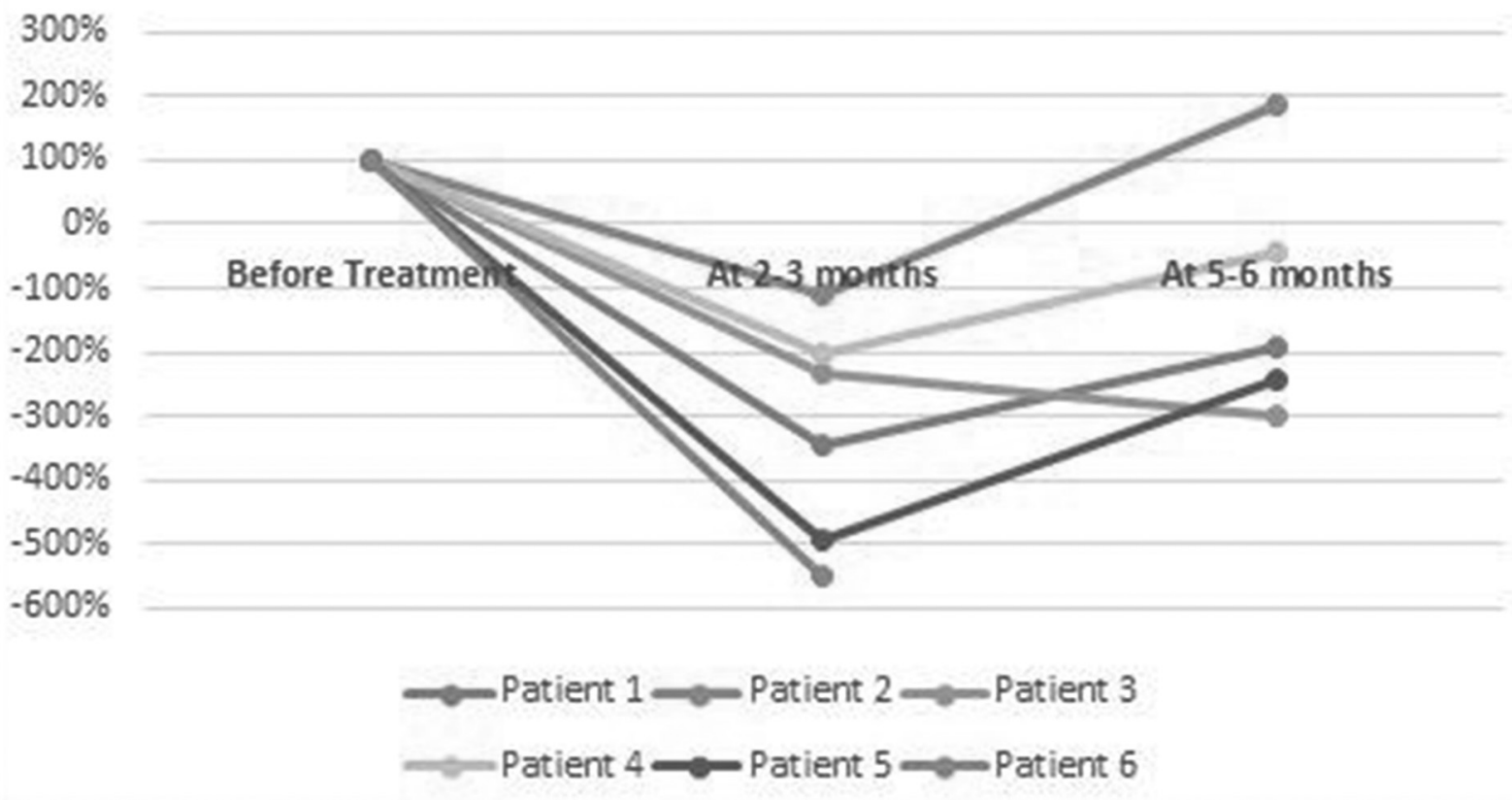

Abstract 95 Figure 1 Changes in the RAIL score from baseline, at 2-3 months and at 5-6 months in 6 patients with LN. Baseline value is assigned $100 \%$

Methods Urine samples were collected from active LN patients prior to induction treatment for $\mathrm{LN}$ and serially afterwards, coinciding with clinical visits. Luminex Bead Multiplex Assay was used for the analyses of urine biomarkers included in the RAIL score (neutrophil gelatinase-associated lipocalin, ceruloplasmin, monocyte chemoattractant protein-1, adiponectin, hemopexin, kidney injury molecule-1). RAIL scores were calculated per the defined algorithm for each urine sample. Data collected include LN histologic classification (International Society of Nephrology (ISN)/Renal Pathology Society (RPS) classification), renal SLE disease activity index (rSLEDAI) score and type of therapy.

Results At the time of the analysis, data from 6 active LN patients were collected longitudinally. Patients were all females and all had class IV LN per the ISN/RPS. Renal SLEDAI scores were on the higher end $(M=11.3, S D=3.9)$. All patients were started on intravenous methylprednisolone and cyclophosphamide (CYC) therapy. All but one patient completed 6 doses of monthly CYC before switching to oral mycophenolate mofetil therapy. The RAIL scores for the 6 patients ranged between -1.8 and 3.29. All patients had reductions in their RAIL score at 2-3 months period at an average of $322 \%$ decline from baseline (Figure 1). At the end of induction treatment or at the 5-6 months interval, 5/6 samples were available for analysis and showed that $4 / 5$ patients maintained a decline of RAIL scores below the baseline. Of note the patient with higher RAIL score at the end of treatment had only 3 monthly doses of CYC. All rSLEDAI scores decreased between baseline and the 6 months interval. However, one patient with known medication non-adherence had a flare of $\mathrm{LN}$ at the 6 months point leading to increased rSLEDAI.

Conclusions RAIL scores show overall improvement from baseline with LN induction therapy. Lack of improvement was associated with flare of disease. Additional data points and a larger study sample are required to study the ability of the RAIL score to reflect clinical improvement of LN.
Funding Source(s): Academic Research Committee of the Cincinnati Childrens Research Foundation.

\section{CEREBRAL HEMODYNAMICS AND MICROCIRCULATORY FUNCTION IN SWEDISH PATIENTS WITH SYSTEMIC LUPUS ERYTHEMATOSUS}

Christopher Sjöwall ${ }^{*}$, Christina Svensson, Hanna Jonasson, Thomas Strömberg, Sara Bergstrand, Per Eriksson, Helene Zachrisson. Linköping University

\subsection{6/lupus-2019-Ism.96}

Background Systemic lupus erythematosus (SLE) is a chronic inflammatory disease characterized by multiple organ involvement. Atherosclerosis is the underlying cause for SLE-related cardiovascular disease, and reliable non-invasive methods for early detection of vascular involvement, including microcirculatory assessment, is important. The aim of this study was to detect whether macro- and microcirculation is impaired in patients with SLE.

Methods Fifteen patients classified with SLE according to the 2012 SLICC criteria (mean age 51.4 years) with moderate atherosclerotic ultrasound findings in common carotid artery, and 15 age- and sex-matched and plaque-free population controls, (mean age 51.7) were investigated. Intima-media thickness (IMT) was recorded with high frequency ultrasound (GE Logic E9) in carotid and central arteries. Microcirculatory oxygen saturation and endothelial function were assessed with EPOS (Enhanced Perfusion and Oxygen Saturation) (PeriFlux 6000, Perimed, Järfälla, Sweden) and EndoPATTM2000 system (Itamar Medical, Israel). The EPOS system measures red blood cell tissue fraction, speed resolved perfusion and oxygen saturation in the microcirculation of the skin. EndoPAT 2000 records changes in finger arterial pulsatile volume reflecting microcirculatory endothelial function. Cerebrovascular reserve capacity was assessed by Transcranial Doppler (TCD) (Sonara TCD (Natus) by detecting mean flow velocities in middle 
cerebral artery at base-line and after $30 \mathrm{~s}$ of breath holding. A breath-hold-index $(\mathrm{BHI})$ of $<0.69$ indicates impaired cerebrovascular reserve capacity.

Results IMT in the aortic arch was higher in patents versus controls, $1.3 \pm 0.3$ vs $1.1 \pm 0.2 \mathrm{~mm}(p=0.04)$, whereas no difference was found in the common carotid artery, 0.61 \pm 0.13 vs $0.55 \pm 0.10 \mathrm{~mm} \quad(p=0.2)$. BHI-values were lower in the SLE-group, $1.29 \pm 0.36$ vs $1.65 \pm 0.56 \quad(p=0.05)$, whereas both groups had signs of preserved cerebrovascular reserve capacity. Mean oxygen saturation peak was decreased in SLE patients versus controls, $79.5 \pm 7.8 \%$ vs $86.9 \pm 5.6 \% \quad(p=0.006)$. Endothelial function using EndoPAT did not differ, 0.72 \pm 0.40 vs $0.84 \pm 0.24,(p=0.3)$.

Conclusions This study indicates that microcirculatory vessel disease, as measured with EPOS and breath-hold index, could be present in SLE cases with atherosclerotic findings. However, the impaired microcirculation in SLE compared to population controls needs further validation in larger patient groups, also including non-atherosclerotic cases with SLE.

Funding Source(s): This work was supported by grants from the Swedish Rheumatism Association, the County Council of Östergötland, the Swedish Society of Medicine, the King Gustaf Vs 80 year anniversary foundation and the King Gustaf V and Queen Victorias Freemasons foundation.

\section{DYNAMIC DIFFUSE OPTICAL SPECTROSCOPY CAN DIAGNOSE AND QUANTIFY LUPUS ARTHRITIS}

${ }^{1}$ George Danias*, ${ }^{2}$ Youngwan Kim, ${ }^{2}$ Alessandro Marone, ${ }^{1}$ Kayla Neville, ${ }^{1}$ Tommy Chen, ${ }^{1}$ Andrea Frantz, ${ }^{1}$ Teja Kapoor, 'Laura Geraldino-Pardilla, ${ }^{2}$ Andreas Hielscher, ${ }^{2}$ Anca D Askanase. ${ }^{1}$ Columbia University Medical Center; ${ }^{2}$ Columbia University

\subsection{6/lupus-2019-Ism.97}

Background SLE arthritis is difficult to evaluate because of the sometimes-evanescent nature of the symptoms and limitations of physical exams and imaging studies. Dynamic diffuse optical spectroscopy (dDOS) can be used to assess changes in light absorption through tissues during transient venous occlusion. The optical signal reflects changes in blood perfusion and has diagnostic value in rheumatoid arthritis. The current study explored the use of dDOS in SLE arthritis.

Methods 12 SLE patients (ACR criteria) with active arthritis and 5 controls were evaluated. A dDOS sensor module was developed (figure. 1a). Hemodynamic effects were obtained by inflating a BP cuff to $40 \mathrm{mmHg} \times 60 \mathrm{~s}$. Light at 3 wavelengths (lambda $=530,655,940 \mathrm{~nm})$ was used to illuminate joints at 8 different points. Transmitted light intensities were measured with Si-photodetectors at 8 other positions (total $8 \times 8 \times 3=192$ signal traces). Swollen, tender and healthy joints were examined by the same assessor.

Results SLE patients and normal controls dDOS data were available for analysis from 66 and 24 proximal interphalangeal (PIP) joints, respectively (PIPs 2-4). Best results were obtained at $530 \mathrm{~nm}$ with cuff inflation at $40 \mathrm{mmHg}$. A representative measurement of 3 SLE arthritis and 3 normal joints is shown in figure $1 \mathrm{~b}$, highlighting differences in rise and plateau time. Given the pronounced effects at lambda $=530 \mathrm{~nm}$, we speculate that altered vessel physiology paired with already-increased blood pooling in the affected

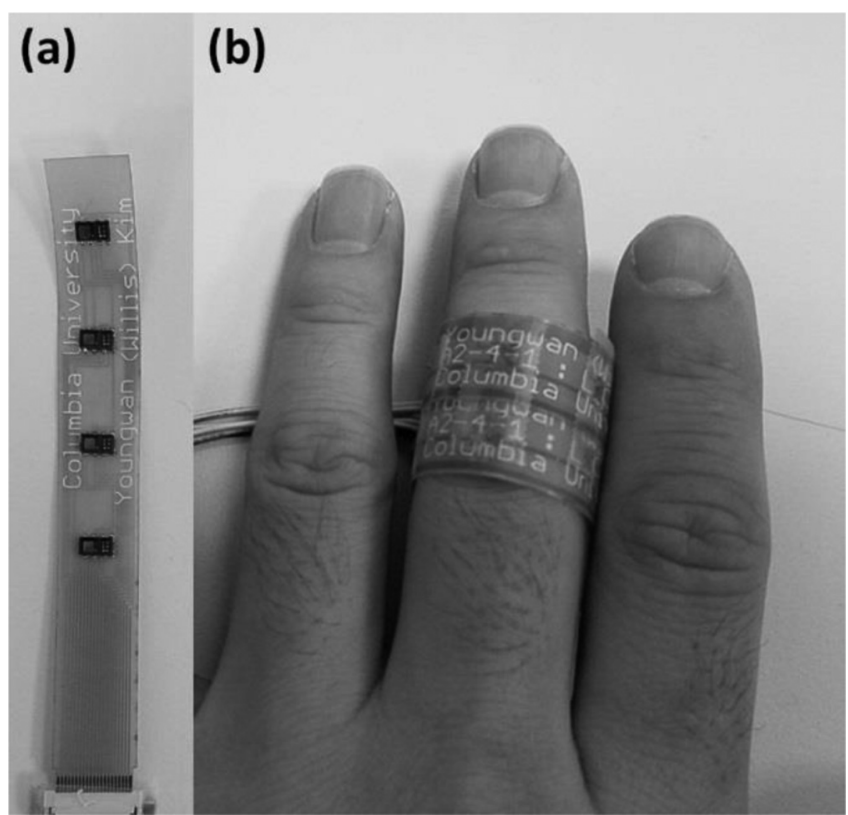

Abstract 97 Figure 1 a) Two dDOS sensor module bands (1a) wrapped around a PIP joint for measurement (1b). Each band contains 4 measurement heads (black dots). Each of the measurement heads includes 3 light-emitting diodes at three different wavelengths $(\lambda=530$ $\mathrm{nm}, 655 \mathrm{~nm}$, and $940 \mathrm{~nm}$, power $=2 \mathrm{~mW}$ ) and one Si-photodetector.

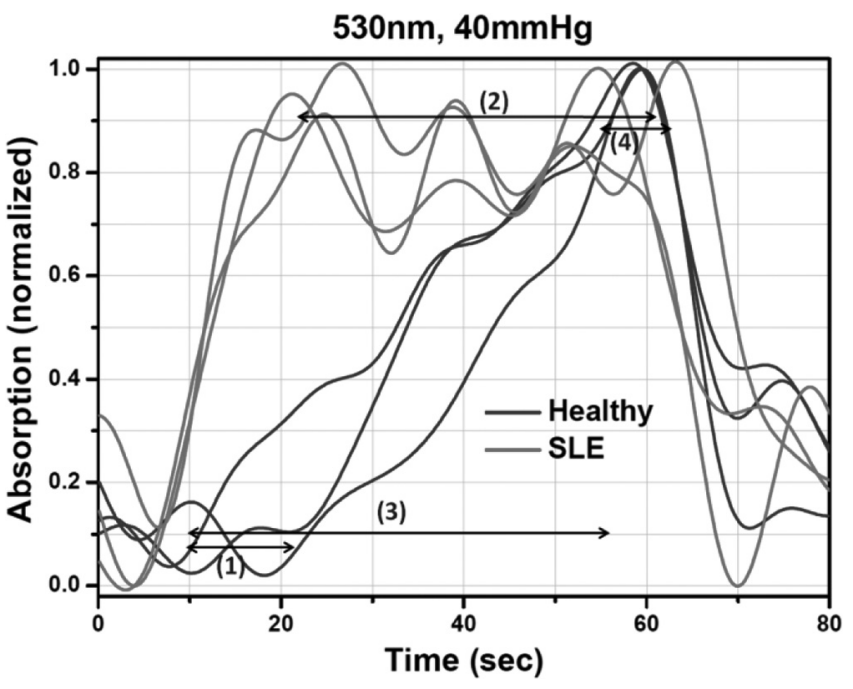

Abstract 97 Figure 1b Representative raw data for one healthy subject (blue) and one SLE arthritis subject (red). (1) and (2) are respectively the rise and the plateau times for a SLE patient, while (3) and (4) are respectively the rise and the plateau times for a healthy

inflamed joints resulted in quicker increase in light absorption (rise time) that is maintained longer (plateau time) compared to normal joints. The AUC for dDOS was consistent with excellent discrimination, $\mathrm{AUC}=0.8639$, sensitivity $=76.19$, specificity $=88.57$ (figure $1 \mathrm{c}$ ).

Conclusions dDOS can evaluate SLE arthritis with high sensitivity and specificity. Rise and plateau time of the optical traces correlate strongly with swollen and tender joint count. The advantages of dDOS are non-invasiveness, objectivity 